\title{
Engineering of vitamin prototrophy in Clostridium ljungdahlii and Clostridium autoethanogenum
}

\author{
Florence J. Annan ${ }^{1} \cdot$ Bakir Al-Sinawi ${ }^{2} \cdot$ Christopher M. Humphreys $^{1} \cdot$ Rupert Norman ${ }^{1} \cdot$ Klaus Winzer $^{1}$. \\ Michael Köpke $^{3} \cdot$ Sean D. Simpson ${ }^{3} \cdot$ Nigel P. Minton $^{1} \cdot$ Anne M. Henstra ${ }^{1}$ (D
}

Received: 26 September 2018 / Revised: 19 February 2019 / Accepted: 12 March 2019 / Published online: 10 April 2019

(C) The Author(s) 2019

\begin{abstract}
Clostridium autoethanogenum and Clostridium ljungdahlii are physiologically and genetically very similar strict anaerobic acetogens capable of growth on carbon monoxide as sole carbon source. While exact nutritional requirements have not been reported, we observed that for growth, the addition of vitamins to media already containing yeast extract was required, an indication that these are fastidious microorganisms. Elimination of complex components and individual vitamins from the medium revealed that the only organic compounds required for growth were pantothenate, biotin and thiamine. Analysis of the genome sequences revealed that three genes were missing from pantothenate and thiamine biosynthetic pathways, and five genes were absent from the pathway for biotin biosynthesis. Prototrophy in $C$. autoethanogenum and C. ljungdahlii for pantothenate was obtained by the introduction of plasmids carrying the heterologous gene clusters pan $B C D$ from Clostridium acetobutylicum, and for thiamine by the introduction of the thiC-purF operon from Clostridium ragsdalei. Integration of pan $B C D$ into the chromosome through allele-coupled exchange also conveyed prototrophy. $C$. autoethanogenum was converted to biotin prototrophy with gene sets bioBDF and bioHCA from Desulfotomaculum nigrificans strain CO-1-SRB, on plasmid and integrated in the chromosome. The genes could be used as auxotrophic selection markers in recombinant DNA technology. Additionally, transformation with a subset of the genes for pantothenate biosynthesis extended selection options with the pantothenate precursors pantolactone and/or beta-alanine. Similarly, growth was obtained with the biotin precursor pimelate combined with genes bioYDA from $C$. acetobutylicum. The work raises questions whether alternative steps exist in biotin and thiamine biosynthesis pathways in these acetogens.
\end{abstract}

Keywords Biosynthetic pathway $\cdot$ Allele coupled exchange $\cdot$ Pantothenate $\cdot$ Thiamine $\cdot$ Biotin $\cdot$ Gas fermentation

\section{Introduction}

Carboxydotrophic acetogenic Clostridium spp. are well placed to play important roles in carbon capture and production of fuels and platform chemicals from various wastes and renewable resources by synthesis gas fermentation (Marcellin

Electronic supplementary material The online version of this article (https://doi.org/10.1007/s00253-019-09763-6) contains supplementary material, which is available to authorized users.

Anne M. Henstra

anne.henstra@nottingham.ac.uk

1 BBSRC/EPSRC Synthetic Biology Research Centre, School of Life Sciences, University of Nottingham, Nottingham NG7 2RD, UK

2 University of New-South Wales (UNSW) Sydney, Kensington, Australia

3 LanzaTech Inc., 8045 Lamon Avenue, Suite 400, Skokie, IL, USA et al. 2016; Liew et al. 2016; Dürre 2017; Humphreys and Minton 2018). The best studied members of this group are the closely related Clostridium autoethanogenum and Clostridium ljungdahlii, and genome scale metabolic models are available for these strains (Bruno-Barcena et al. 2013; Marcellin et al. 2016; Nagarajan et al. 2013). Many Clostridium spp. are fastidious microorganisms and are by default grown in complex media. This is also true for C. autoethanogenum and C. ljungdahlii. Use of defined media avoids interference of unspecified organic compounds present in complex media, potentially reduces cost of the medium, helps to reduce risk of infection of cultures and improves process exergy (Dadak et al. 2016; Aghbashlo et al. 2016). Fastidious microorganisms require the addition of organic molecules for which they are auxotrophic to support their growth. These are often specific amino acids or vitamins, for which their biosynthesis pathway is incomplete. Heterologous genes for absent metabolic steps can be used as selectable 
markers to ensure the stable maintenance of recombinant plasmids carrying a desirable trait. Traditionally, such plasmids are maintained through incorporation into the plasmid of genes that confer resistance against antibiotics or other toxic compounds and the addition of these toxic compounds to the growth media. The presence of antibiotics can affect cellular function, while their cost can preclude their use in large-scale processes. Moreover, in an era of increasing antimicrobial resistance, the use of such genes should be avoided (Pronk 2002).

Genetic tools are available for markerless integration of heterologous genes into the genomes of various Clostridium spp. (Minton et al. 2016). This creates the opportunity to develop stable prototrophic strains of fastidious microorganisms once it is known which genes are needed through their integration into the chromosome. Of particular utility is the method allele-coupled exchange (ACE), which can be used to introduce DNA cargo of any size or complexity at defined genomic loci, most commonly pyrE, a pivotal gene involved in uracil biosynthesis (Heap et al. 2012). The selection used is dependent whether the targeted pyrE allele is mutant or wild type. In the most useful configuration, the former is employed and the desired payload is integrated into the chromosome concomitant with restoration of the cell to uracil prototrophy, a readily selectable phenotype. The use of ACE to both create pyrE deletions and thereafter correct back to wild type was recently demonstrated in $C$. autoethanogenum using pyrE (Liew et al. 2017). Insertion of genes into the chromosomes of C. autoethanogenum or C. ljungdahlii by $\mathrm{ACE}$ is yet to be demonstrated.

Here, we investigate auxotrophy in $C$. autoethanogenum and C. ljungdahlii, identify the genes that are needed to create prototrophy for pantothenate, biotin and thiamin and transform these microorganisms with heterologous genes to prototrophy. Removing auxotrophies will allow growth on completely defined media without any other carbon sources other than gas/sugar and benefit systems biology studies. Also, removal of vitamin supplementation would lower process costs as growth medium makes up a significant part of costs of an industrial process. The ability of C. autoethanogenum transformed with the bioYDE gene cluster of $C$. acetobutylicum to grow when pimelic acid is added to the medium suggests that unknown pathway steps exist for BioW and BioF in biotin synthesis. Similarly, the apparent lack of thiG and thiH did not prevent growth of strains transformed with the thiC-purF operon from Clostridium ragsdalei in the absence of thiamine and suggests the presence of alternatives. Additionally, the reliance on $\operatorname{panB}$, panC and panD and low availability of pantothenate in yeast extract allow multifactorial or iterative plasmid-based genetic engineering in combination with the use of pantolactone and beta-alanine as a tool.

\section{Materials and methods}

\section{Strains}

Clostridium autoethanogenum DSM10061 (Abrini et al. 1994), Clostridium ljungdahlii DSM13528 (Tanner et al. 1993) and Clostridium ragsdalei DSM15248 were obtained from the Deutsche Sammlung von Mikroorganismen und Zellkulturen (DSMZ, Germany). Clostridium acetobutylicum ATCC 824 was kindly provided by H. Bahl (University of Rostock, Germany). E. coli CA434 (Purdy et al. 2002) was kindly provided by M. Young (University College Wales, Aberystwyth, UK).

\section{Growth conditions}

Bacterial stocks were kept at $-80{ }^{\circ} \mathrm{C}$ in medium with $15 \%$ $v / v$ DMSO or $25 \% v / v$ glycerol as cryoprotectant and routinely (pre-)grown at $37^{\circ} \mathrm{C}$ in YTF medium (composition below) or for $E$. coli in $\mathrm{LB}$ medium (in $\mathrm{g} / \mathrm{L}$ : yeast extract (YE), 5; tryptone, $10 ; \mathrm{NaCl}, 10)$. Solid media contained $15 \mathrm{~g} / \mathrm{L}$ bacteriological agar (Oxoid) in addition to the components of the liquid medium. Cultures of Clostridium strains were grown anaerobically in a MG1000 workstation equipped with TG airlock (Don Whitley Scientific, UK) supplied with $\mathrm{N}_{2}, \mathrm{CO}_{2}$ and $\mathrm{H}_{2}$ or a PureLab glovebox system (Inert, Amesbury, MA, USA) or when CO was used, in glass serum bottles that were sealed with butyl rubber stopper (Rubber B.V., Netherlands) inside the anaerobic workstation. A complex medium (YTF medium), or a defined 2-(N-morpholino)-ethanesulfonic acid (MES) buffered mineral medium (MBM medium) with vitamins, was used. YTF medium contained per litre: yeast extract, $10 \mathrm{~g}$; tryptone, $16 \mathrm{~g}$; fructose, $10 \mathrm{~g}$; sodium chloride, $0.2 \mathrm{~g}$; and $1 \mathrm{~mL}$ vitamin solution (see below). MBM medium, after Rajagopalan et al. (2002), contained (per L) $5.0 \mathrm{~g}$ MES, pH 5.8; $25 \mathrm{~mL}$ macronutrient solution (MNS); $10 \mathrm{~mL}$ trace element solution (TES); and $1 \mathrm{~mL}$ vitamin solution. MNS contained (in g/L) $\mathrm{NH}_{4} \mathrm{Cl}, 40 ; \mathrm{KH}_{2} \mathrm{PO}_{4}$, 8.0; $\mathrm{MgSO}_{4} \cdot 7 \mathrm{H}_{2} \mathrm{O}, 8.0 ; \mathrm{KCl}, 4.0 ; \mathrm{CaCl}_{2}, 0.8$; and $\mathrm{NaCl}$, 32. TES contained (in $\mathrm{g} / \mathrm{L}$ ) nitrilotriacetic acid, $2 ; \mathrm{MnSO}_{4}$. $\mathrm{H}_{2} \mathrm{O}, 1 ; \mathrm{Fe}\left(\mathrm{SO}_{4}\right)_{2}\left(\mathrm{NH}_{4}\right)_{2} \cdot 6 \mathrm{H}_{2} \mathrm{O}, 0.8 ; \mathrm{CoCl}_{2} \cdot 6 \mathrm{H}_{2} \mathrm{O}, 0.2$; $\mathrm{ZnSO}_{4} \cdot 7 \mathrm{H}_{2} \mathrm{O}, 0.02 ; \mathrm{CuCl}_{2} \cdot 2 \mathrm{H}_{2} \mathrm{O}, 0.02 ; \mathrm{NaMoO}_{4} \cdot 2 \mathrm{H}_{2} \mathrm{O}$, $0.02 ; \mathrm{Na}_{2} \mathrm{SeO}_{3}, 0.02 ; \mathrm{NiCl}_{2} \cdot 6 \mathrm{H}_{2} \mathrm{O}, 0.02$; and $\mathrm{Na}_{2} \mathrm{WO}_{4}$. $2 \mathrm{H}_{2} \mathrm{O}, 0.02$. The vitamin solution contained (in $\mathrm{mg} / \mathrm{L}$ ) thiamine $\mathrm{HCl}, 50$; riboflavin, 50; nicotinic acid, 50; calcium pantothenate, 50; pyridoxine- $\mathrm{HCl}, 10$; biotin, 20; folic acid, 20; 4-aminobenzoic acid, 50; cyanocobalamin, 50; and lipoic acid, 50. Fructose $(40 \mathrm{mM})$ was used as growth carbon and energy source, except when $\mathrm{CO}\left(\mathrm{P}_{\mathrm{CO}}=\right.$ $100 \mathrm{kPa}$ ) was used, which was added to the existing serum bottle gas phase. L-cysteine $(1 \mathrm{~g} / \mathrm{L})$ served as reducing agent and source of sulfur, except when sodium sulfide 
(2.5 mM) was used. The following supplements were added to or omitted from MBM medium as indicated: YE $(10 \mathrm{~g} / \mathrm{L})$, vitamin-free casein acid hydrolysate (CAS, $10 \mathrm{~g} /$ L) (Sigma), 5-fluoroorotic acid (FOA, $500 \mathrm{mg} / \mathrm{L}$ ), uracil $(\mathrm{U}, 1 \mathrm{mg} / \mathrm{L})$, pantolactone $(50 \mu \mathrm{g} / \mathrm{L})$, beta-alanine $(50 \mu \mathrm{g} / \mathrm{L})$ and pimelic acid $(20 \mu \mathrm{g} / \mathrm{L})$. Antibiotics were used when indicated, at following concentrations: clarithromycin $(\mathrm{Cl}), 6 \mathrm{mg} / \mathrm{L}$; thiamphenicol (Tm), $7.5 \mathrm{mg} / \mathrm{L}$; erythromycin (Em), $500 \mathrm{mg} / \mathrm{L}$; chloramphenicol $(\mathrm{Cm}), 25 \mathrm{mg} / \mathrm{L}$.

\section{Phenotypic analysis}

Growth of C. ljungdahlii and C. autoethanogenum in medium lacking one or more sources of amino acids or vitamins was tested as indicated (Table 1) to identify essential amino acids and vitamins needed for growth. The test was carried out in $25-\mathrm{mL}$ Hungate tubes that contained $20 \mathrm{~mL}$ of medium with inoculation volume of $1 \% \mathrm{v} / \mathrm{v}$ over 5 serial transfers and $48 \mathrm{~h}$ between serial transfers to allow the culture to grow. The optical densities (OD) of cultures in the Hungate tubes were recorded using a biowave CO8000 portable cell density meter (Biochrom, UK). Qualitative growth was indicated by an increase in OD and confirmed by presence of cells of the correct morphology in the fifth dilution by phase contrast microscopy (Nikon Eclipse Ci). The initial inoculum for the serial transfer was a culture in exponential growth phase, grown in $\mathrm{MBM}+\mathrm{CAS}$ (MBM with CAS added), cysteine and full vitamin complement added. Requirement of amino acids for growth was tested in MBM medium with sodium sulfide and full vitamin complement added. Requirement of vitamins for growth was tested in MBM medium supplemented with CAS, cysteine and a vitamin solution lacking one of each of the vitamins.

\section{Genotypic analysis}

Completeness of metabolic pathways for vitamin biosynthesis in C. autoethanogenum and C. ljungdahlii was assessed using the published whole genome metabolic models iHN637 and iCLAU786 (Nagarajan et al. 2013; Marcellin et al. 2016) and KEGG pathway database information (Kanehisa et al. 2017). Sequences to validate identified genes, or of orthologs of missing genes in incomplete pathways, were obtained from KEGG or UNIPROT databases (Bateman et al. 2017) and used in BLAST (Altschul et al. 1990) provided by NCBI (Agarwala et al. 2017). Whole genome sequences of C. autoethanogenum with GenBank accession numbers GCA_000484505.1, GCA_001484725 and C. ljungdahlii GCA 000143685.1 were used (Brown et al. 2014; Humphreys et al. 2015; Köpke et al. 2010).

\section{Molecular genetic tools}

All used enzymes were from New England Biolabs (NEB), gel extraction and plasmid miniprep kits were from Qiagen, Zymo Research or NEB, and the manufacturer's protocols were followed. PCR primers were synthesised by Sigma, Eurofins Genomics or LifeTechnologies (Table 2).

Genomic DNA of Desulfotomaculum nigrificans CO-1SRB (Parshina et al. 2005; Visser et al. 2014) was obtained from DSMZ (DSM14880). The DNeasy blood and tissue kit (Qiagen) were used to isolate genomic DNA from C. acetobutylicum. Genomic DNA of $C$. ragsdalei was extracted as follows. A $100-\mathrm{mL}$ sample of an exponentially growing culture was harvested $\left(4000 \times g, 15 \mathrm{~min}, 4{ }^{\circ} \mathrm{C}\right)$, washed with potassium phosphate buffer $(10 \mathrm{mM}, \mathrm{pH} 7.5)$ and re-suspended in 1.9-mL STE buffer $(50 \mathrm{mM}$ Tris- $\mathrm{HCl}$, pH 8.0, 1 mM EDTA, $200 \mathrm{mM}$ sucrose). The cells were incubated for $30 \mathrm{~min}$ with $300 \mu \mathrm{L}$ of lysozyme $(100,000 \mathrm{U})$ at $37^{\circ} \mathrm{C}$. The lysis step was followed by a 10 -min incubation with $10 \%(w / v)$ SDS. The RNA was digested at room temperature by adding $240 \mu \mathrm{L}$ of $0.5 \mathrm{M}$ EDTA (pH 8.0), $20 \mu \mathrm{L}$ of $1 \mathrm{M}$ Tris- $\mathrm{HCl}(\mathrm{pH} 7.5)$ and $100 \mu \mathrm{L}$ of RNase A. Then, $100 \mu \mathrm{L}$ proteinase $\mathrm{K}(0.5 \mathrm{U})$ were added and proteolysis took place for $3 \mathrm{~h}$ at $37^{\circ} \mathrm{C}$. Finally, $600 \mu \mathrm{L}$ of sodium perchlorate $(5 \mathrm{M})$ was added, followed by a phenol-chloroform extraction and an isopropanol precipitation.

Sanger sequencing was used to validate DNA sequences using plasmid or PCR products as template (by Eurofins Genomics or Source Bioscience). Plasmids used in this study are listed in Table 3.

\section{Plasmid DNA transfer into Clostridium spp.}

Plasmids were introduced into $C$. autoethanogenum by conjugation from the E. coli donor strain CA434 as previously described (Liew et al. 2017). In contrast, plasmids were introduced into $C$. ljungdahlii by electroporation as follows. Electrocompetent cells of $C$. ljungdahlii were prepared from a culture grown in $300-\mathrm{mL}$ YTF medium in exponential growth phase with an OD600 of 0.2-0.3. Cells were pelleted and washed twice in $30-\mathrm{mL}$ anaerobic SMP5.8 buffer (1 mM sodium phosphate $\mathrm{pH} 5.8,1 \mathrm{mM}$ $\mathrm{MgCl}_{2}, 270 \mathrm{mM}$ sucrose) in anaerobic $50-\mathrm{mL}$ conical polypropylene tubes using an 5430R centrifuge (Eppendorf, Germany) placed inside a model B vinyl anaerobic chamber (Coy Laboratory Products, MI, USA) at $4552 \times g$ for $5^{\prime}$ at ambient temperature. Cell pellets were resuspended by vortexing and then pooled. After the second wash, the cell pellet was resuspended in $1.8-\mathrm{mL}$ SMP5.8 buffer and $0.2 \mathrm{~mL}$ DMSO, divided over $2-\mathrm{mL}$ polypropylene screw cap microtubes (Sarstedt) in $0.11-\mathrm{mL}$ aliquots, flash frozen in liquid nitrogen and stored at $-80{ }^{\circ} \mathrm{C}$. For transformation of electrocompetent cells 


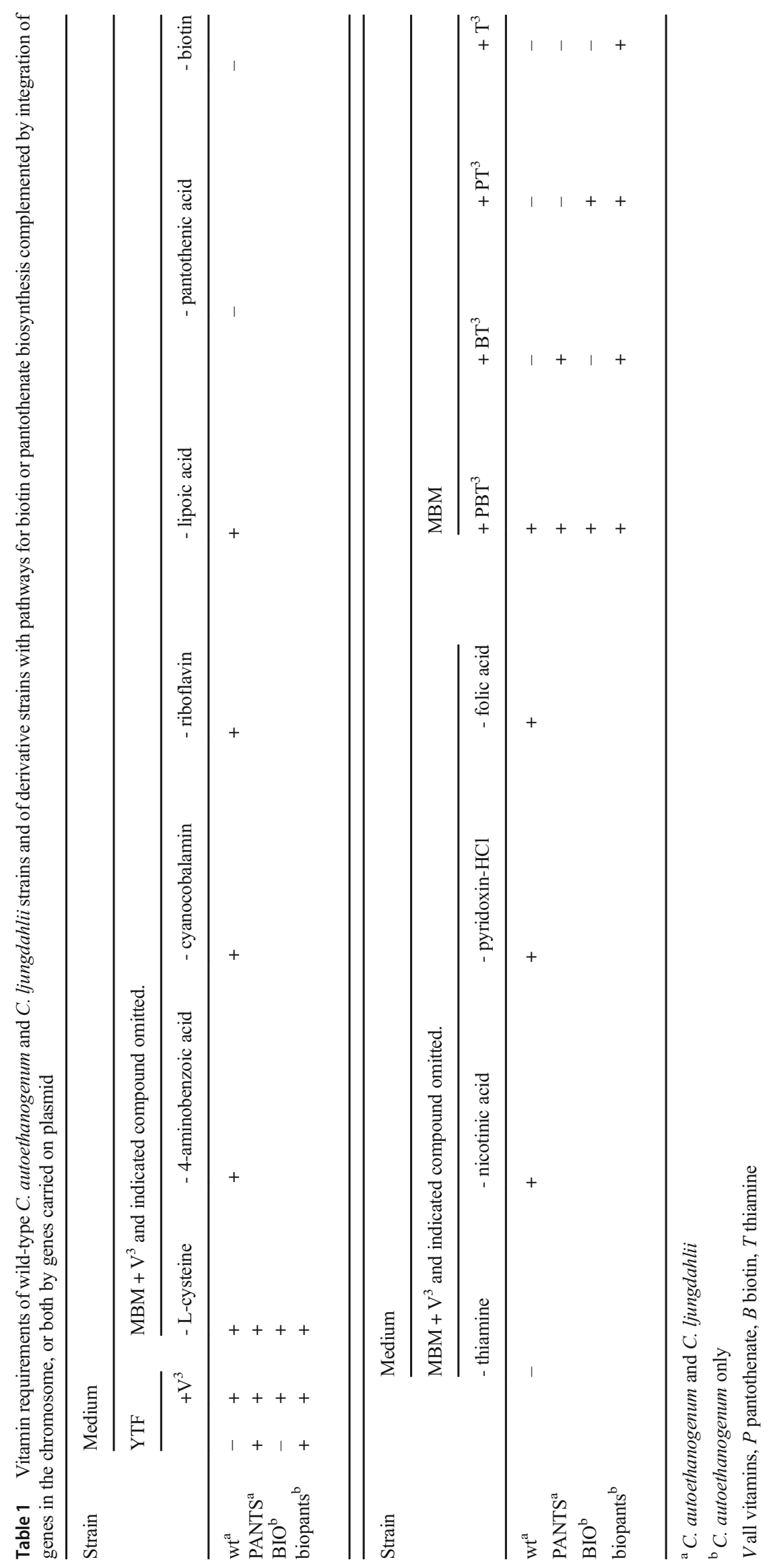


Table 2 Primers used in this study

\begin{tabular}{|c|c|c|}
\hline Primer ID & Sequence & Endonuclease/template \\
\hline \multicolumn{3}{|l|}{ Pantothenate } \\
\hline panBfwd & tatacatatgaaaaataccactgaaac & NdeI/C. acetobutylicum \\
\hline panCfwd & tatacatatggaaattttgcattcc & NdeI/C. acetobutylicum \\
\hline panDfwd & tatacatatgcatttaaatatgttaaaatctaaa & NdeI/C. acetobutylicum \\
\hline panBrev & tatagctagcttatttggtaatttcgtctataatg & NheI/C. acetobutylicum \\
\hline panCrev & tatagctagcttatatattccaagtgaaattatctataagg & NheI/C. acetobutylicum \\
\hline panDrev & Tatagctagcttagccaattgtcc & NheI/C. acetobutylicum \\
\hline panB2fwd & Tataactagtatgaaaaataccactgaaac & SpeI/C. acetobutylicum \\
\hline \multicolumn{3}{|l|}{ Biotin } \\
\hline bioYDAF1 & tatacatatgattatggtttctattttgc & NdeI/C. acetobutylicum \\
\hline bioYDAR1 & tatagctagcttattcaagatactgctctatactttc & NheI/C. acetobutylicum \\
\hline bioBDFF1 & tatacatatgagcaatattttgaaacatatcg & NdeI/D. carboxidivorans \\
\hline bioBDFR1 & tatagctagcttaaatcaatcccagttc & NheI/D. carboxidivorans \\
\hline bioHCAF1 & tatacatatgttggaaaaacagagattaatcc & NdeI/D. carboxidivorans \\
\hline bioHCAR1 & tatagctagcttaatctattccaaaatagttattaattgc & NheI/D. carboxidivorans \\
\hline \multicolumn{3}{|l|}{ Thiamine } \\
\hline thiCpurFfwd & gcagggcccaatacgattatctcctttc & ApaI/C. ragsdalei \\
\hline thiCpurFrev & gcatcctgcaggtaaattttgttcttcatt & Sbfl/C. ragsdalei \\
\hline \multicolumn{3}{|l|}{$\mathrm{ACE}$} \\
\hline SHAF & tccgcggaagggcattttctactttcttc & SacII/C. ljungdahlii \\
\hline SHARd & agcggccgecacatcttcagaaattataattttttctcc & Not $\mathrm{I} / C$. ljungdahlii \\
\hline SHARc & agcggccgcttatttgaatactctacttccaggc & Not $\mathrm{I} / C$. ljungdahlii \\
\hline GHAF & tatgctagcgtcgaaaaaatcaatgcacgatgc & NheI/C. ljungdahlii \\
\hline GHAR & aggcgcgectactgggtaacggaacctg & AscI/C. ljungdahlii \\
\hline $\mathrm{CH} 21 \mathrm{th} / \mathrm{F} 1$ & tatagctagcccgcttttaacaaaatatattg & NheI/pMTL8xxx2 \\
\hline $\mathrm{CH} 21$ thlR1 & tataactagtggggtaacagataaaccatttc & SpeI/pMTL8xxx2 \\
\hline cacpyreF & atggaacaatacaaacaagaatttatag & / C. acetobutylicum \\
\hline cacpyreR & gagtactataaagagtatggagtaaaatag & / C. acetobutylicum \\
\hline \multicolumn{3}{|l|}{ HiFi assembly } \\
\hline P1F & atgaaatagattgaaatgagcaatattttgaaacatatc & / pMTL-FJAL22 \\
\hline $\mathrm{P} 2 \mathrm{R}$ & gctactacacttaaatcaatcccagttcttttc & / pMTL-FJAL22 \\
\hline $\mathrm{P} 3 \mathrm{~F}$ & ggattgatttaagtgtagtagcetgtgaaataag & / pMTL-FJAL23 \\
\hline $\mathrm{P} 4 \mathrm{R}$ & ttgatttttcgacgctagcttaatctattccaaaatagttattaattgc & / pMTL-FJAL23 \\
\hline
\end{tabular}

$100-\mu \mathrm{L}$ suspension was transferred to a 2-mm electroporation cuvette (Biorad) with $10-\mu \mathrm{L}$ plasmid solution and pulsed $(1000 \mathrm{~V}, 50 \mu \mathrm{F}, 200 \mathrm{Ohm})$ with a GenePulser Xcell electroporation instrument equipped with $\mathrm{PC}$ and CE modules (Biorad). The pulsed cell suspension was transferred to 5-mL anaerobic YTF recovery medium, incubated for $20 \mathrm{~h}$ and pelleted. Cell pellets were resuspended in $100-\mu \mathrm{L}$ spent recovery medium and plated on solid YTF medium with appropriate selection marker. Colonies would form over 2-3 days. RCLJU medium was used instead of YTF medium and an EBA21 centrifuge (Hettich, Germany) placed inside a Don Whitley MG1000 anaerobic cabinet for the partial deletion of pyrE in C. ljungdahlii described below in the procedure detailed above. RCLJU medium was composed as MBM medium with YE, except that acid and alkaline trace element solutions (Stams et al. 1993) were used instead.

\section{Construction of expression vectors}

Genes that were identified as necessary to complete biotin or pantothenate metabolic pathways were amplified by PCR with Q5 DNA polymerase, using genomic DNA of donor species as a template. Similarly, genes for completion of thiamine pathway were amplified using iProof HF DNA polymerase (Bio-Rad) and FailSafe $2 \times$ PCR premix E buffer 
Table 3 Plasmids constructed in this study

\begin{tabular}{|c|c|c|c|c|}
\hline Plasmid & Backbone $^{\text {a }}$ & Functional module & F-primer & R-primer \\
\hline \multicolumn{5}{|l|}{ ACE } \\
\hline pANNE98 & pMTL84141 & dSHA & SHAF & SHARd \\
\hline pANNE99 & pMTL84141 & GHA & GHAF & GHAR \\
\hline pMTL-AMH100 & pANNE99 & ACE PyrE deletion & & \\
\hline pMTL-AMH101 & pMTL-CH14 & ACE PyrE deletion & & \\
\hline pMTL-AMH102 & pMTL84141 & ACE PyrE wt-restore & SHAF & GHAR \\
\hline pMTL-AMH103 & pANNE99 & ACE PyrE correction & SHAF & SHARc \\
\hline pMTL-CH14 & pMTL84151 & C. acetobutylicum pyrE ${ }^{\mathrm{b}}$ & cacpyreF & cacpyreR \\
\hline pMTL-CH20 & pMTL84151 & ACE PyrE correction & & \\
\hline pMTL-CH21 & pMTL-CH20 & ACE PyrE correction + BB2 & & \\
\hline pMTL-CH23 & pMTL-CH21 & $\mathrm{ACE}$ PyrE correction $+\mathrm{P}_{\mathrm{thl}}+\mathrm{BB} 2$ & $\mathrm{CH} 21 \mathrm{thlF} 1$ & $\mathrm{CH} 21$ thlR 1 \\
\hline \multicolumn{5}{|l|}{ Pantothenate } \\
\hline pMTL-FJAL3 & pMTL82152 & $\mathrm{P}_{\text {thl }}-$ panBCD & panBfwd & panDrev \\
\hline pMTL-FJAL4 & pMTL83153 & $\mathrm{P}_{\mathrm{fdx}}-\mathrm{panCD}$ & panCfwd & panDrev \\
\hline pMTL-FJAL5 & pMTL83153 & $\mathrm{P}_{\mathrm{fdx}}-\mathrm{panBC}$ & panBfwd & panCrev \\
\hline pMTL-FJAL6 & pMTL82252 & $\mathrm{P}_{\text {thl-panB }}$ & panBfwd & panBrev \\
\hline pMTL-FJAL7 & pMTL82252 & $\mathrm{P}_{\text {thl-panC }}$ & panCfwd & panCrev \\
\hline pMTL-FJAL8 & pMTL82252 & $\mathrm{P}_{\text {thl-panD }}$ & panDfwd & panDrev \\
\hline pMTL-FJAL10 & pMTL-CH23 & $\mathrm{P}_{\text {thl }}-$ panBCD & panB2fwd & panDrev \\
\hline \multicolumn{5}{|l|}{ Biotin } \\
\hline pMTL-FJAL20 & pMTL82252 & $\mathrm{P}_{\text {thl-bioYDA }}$ & bioYDAF1 & bioYDAR1 \\
\hline pMTL-FJAL21 & pMTL82252 & $\mathrm{P}_{\text {thl }}$-bioBDF & bioBDFF1 & bioBDFR1 \\
\hline pMTL-FJAL22 & pMTL83153 & $\mathrm{P}_{\mathrm{fdx}}-\mathrm{bioHCA}$ & bioHCAF1 & bioHCAR1 \\
\hline pMTL-FJAL23 & pMTL-CH23 & $\mathrm{P}_{\mathrm{thl}}-$ bioBDF- $\mathrm{P}_{\mathrm{fdx}}-\mathrm{bioHCA}$ & HiFi assembly & \\
\hline pMTL-FJAL24 & pMTL83251 & $\mathrm{P}_{\text {thl }}-$ bioBDF- $\mathrm{P}_{\mathrm{fdx}}-$ bioHCA & & \\
\hline \multicolumn{5}{|l|}{ Thiamine } \\
\hline pMTL-BAS1 & pMTL85242 & $P_{\text {native }}$-thiC-purF & thiCpurFfwd & thiCpurFrev \\
\hline
\end{tabular}

(Epicentre). PCR products were digested with endonucleases indicated for each primer pair (Table 2), purified by agarose gel electrophoresis, extracted from gel, and subsequently ligated to a pMTL80000 series shuttle vector (Heap et al. 2009) that was digested with the same endonuclease pair and extracted from gel. Ligation products were used to transform $E$. coli TOP10 (Invitrogen), and transformants with plasmid were selected on solid LB media with appropriate antibiotic selection marker. Plasmid DNA was isolated and submitted for Sanger sequencing to validate the nucleotide sequence of the cloned genes. Only expression vector pMTL-FJAL24 was obtained by subcloning a DNA fragment from another plasmid (pMTL-FJAL23, described below) into pMTL83251 using NotI and NheI.

\section{Creation of ACE vectors}

Genes were integrated in the chromosomes in strains of C. autoethanogenum and C. ljungdahlii using ACE in combination with the pyrE allele (Heap et al. 2012). A set of $A C E$ vectors for pyrE deletion, restoration and correction was constructed as depicted (Fig. 1) as follows. The long region of homology (GHA) and the short region of homology needed to create the partial pyrE deletion (dSHA) were amplified by PCR with indicated primers (Table 3 ) and each product ligated into a pMTL84141 vector after digestion with the indicated restriction endonucleases (Table 2) and treatment of the vector with antarctic alkaline phosphatase (AAP). Ligation products were used to transform $E$. coli XL1-blue chemical competent cells (Agilent). Successful transformants carried vector pANNE98 with dSHA or pANNE99 with GHA (Table 3). Both homology regions were combined in one vector after restriction digestion of both vectors with NheI and AscI, extraction of restriction fragments from gel and ligation of the GHA region into pANNE98 backbone. Ligation products were used to transform E. coli XL1-blue. Successful transformants carried vector pMTL-AMH100. The vector pMTL-AMH101 was created after it was found that with 
pMTL-AMH100, plasmid loss was not obtained in the process required to create the partial pyrE gene deletion (data not shown). Vector pMTL-AMH101 contained pyrE of C. acetobutylicum directly downstream of catP on the vector backbone to enforce plasmid loss during 5-FOA selection. To create pMTL-AMH101, the functional module of pMTLAMH100 was subcloned into pMTL-CH14 using Sbfl and AscI. The plasmid pMTL-CH14 itself was obtained after ligating the PCR-amplified pyrE gene of C. acetobutylicum with indicated primers (Table 3 ) in pMTL84151 after digestion with HpaI, transformation of $E$. coli TOP10. In successful pMTL-CH14 transformants, the inserted pyrE was of the same orientation as catP.

The vectors pMTL-AMH102, with native regions of short and long homology adjoined (SHAGHA) to restore the partial chromosomal pyrE deletion to wildtype, and pMTLAMH103, with the short region of homology to correct the partial chromosomal pyrE deletion (cSHA) and integrate a genetic payload at the same time, were also derived from pMTL-AMH100, as follows. The regions of homology were amplified with indicated primers (Table 3 ) by PCR, digested with indicated restriction endonucleases (Table 2) and extracted from gel. The vector pMTL-AMH100 was digested with the same pairs of restriction endonucleases and extracted from gel, before ligation of the processed PCR fragments, and transformation of $E$. coli XL1-blue with ligation products. The functional module of pMTL-AMH103 was then subcloned using SbfI and AscI into pMTL84151 to obtain the vector pMTL-CH20 which was suitable for transconjugation. A promoter to drive expression of a gene payload of the integration vector was introduced in two successive steps. First, a synthetic BioBrick2 (Knight 2003) fragment (topology: 5'-BB2prefix [EcoRI-NotI-SpeI]synthethic_gene-BB2suffix[NheI-NotI-Pst I]) was subcloned in pMTL-CH20 using Not I and NheI, resulting in pMTL$\mathrm{CH} 21$. Then, a PCR product, with $\mathrm{P}_{\text {thl }}$ amplified using indicated primers (Table 3) from a suitable pMTL80000 vector that carried this promoter as template, was digested with SpeI (restriction site originating from the $\mathrm{BB} 2$ prefix) and NheI (compatible to SpeI) and ligated in plasmid pMTL-CH21 that was only digested with SpeI and treated with AAP. Successful E. coli Top10 transformants contained pMTL-CH23.

\section{Creation of partial pyrE deletion strains}

The vector pMTL-AMH101 was used to delete the Cterminus of pyrE in $C$. autoethanogenum and $C$. ljungdahlii as follows. After transformation of $C$. autoethanogenum with pMTL-AMH101, thiamphenicol-resistant clones were restreaked onto solid YTF + FU (FOA and uracil) medium. Colonies that formed were then restreaked on solid MBM medium (without uracil), MBM + U medium (with uracil) and MBM + UTm medium (with uracil and thiamphenicol).
Clones that grew on MBM + U medium but not on MBM and $\mathrm{MBM}+\mathrm{UTm}$ medium were selected for further work. Deletion of the $3^{\prime}$ end of pyrE in the chromosome of obtained clones was validated by isolation of genomic DNA, amplification of pyrE locus by PCR and Sanger sequencing of the PCR product.

After transformation of C. ljungdahlii, thiamphenicolresistant transformants were used to inoculate liquid rCLJU medium (composed as RCLJU except with $1 \mathrm{~g} / \mathrm{L} \mathrm{YE)} \mathrm{with}$ thiamphenicol. From the resultant cultures, $100 \mu \mathrm{L}$ was spread on solid CCLJU + FU medium (composed as RCLJU, except with CAS instead of YE) and colonies that formed were restreaked on solid CCLJU + FU medium. Colonies that then formed were restreaked on solid CCLJU medium (without uracil), solid RCLJU medium and solid RCLJU + Tm medium. Deletion of pyrE was validated for clones that only grew on RCLJU medium (without Tm).

\section{Chromosomal integration of heterologous genes}

Genes were integrated in the chromosomes of C. autoethanogenum $\triangle p y r E$ and C. ljungdahlii $\triangle p y r E$ by ACE. A vector (pMTL-FJAL10) for integration of panBCD was constructed similar to the construction of expression vectors. PCR products were subcloned into pMTL-CH23 using SpeI and NheI. A vector for integration of bioBDF-bioHCA (pMTL-FJAL23) was constructed by HiFi DNA assembly from three DNA fragments (F1, F2 and F3) using four primers (P1F, P2R, P3F and P4R) following manufacturer's instructions (Fig. 2). Fragment F1 was amplified by PCR from pMTL-FJAL21 as template with primers P1F and P2R (Table 2). Fragment F2 was amplified by PCR from pMTLFJAL22 as template with primers P3F and P4R (Table 2). Plasmid pMTL-CH23 digested with NotI and NheI and extracted from agarose gel served as fragment F3. Validated integration vectors (pMTL-FJAL10 and pMTL-FJAL23) were used to transform $\triangle p y r E$ strains and transformants or transconjugants selected on medium with antibiotic and uracil supplemented, then restreaked on medium without uracil and medium with antibiotic in parallel, until antibiotic resistance was lost, to select for chromosomal integrants that lost the integrative plasmid. The DNA base sequence of the integrated heterologous genes was validated.

\section{Results}

\section{Phenotypic analysis}

Growth experiments in various defined media that lacked specific amino acids or vitamins were performed to establish for which compounds $C$. ljungdahlii and $C$. autoethanogenum were auxotrophic. A MES-buffered mineral base medium 

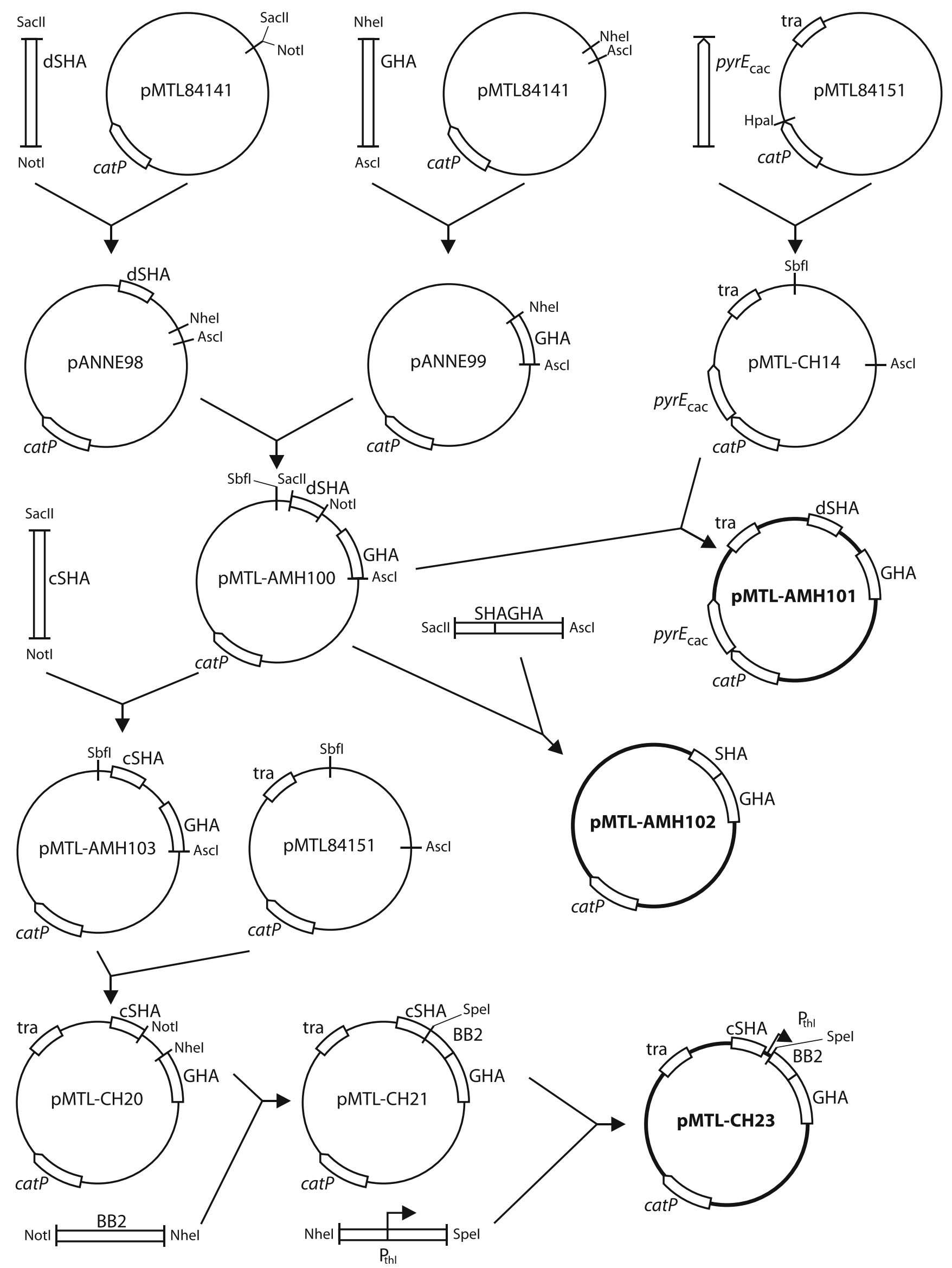
Fig. 1 Schematic representation of subcloning of the ACE vectors pMTL-AMH101 for deletion of pyrE, pMTL-CH23 for correction of pyrE and pMTL-AMH102 for resaturation of the pyrE locus. Full detail is provided in "Material and methods". Linear fragments represent PCR products, except for the BB2 fragment which was synthesised. Subcloning steps used the indicated restriction endonucleases. Sizes of depicted molecules do not relate to molecular size

with vitamins (MBM) to which amino acids, YE or CAS were added, or from which vitamins were omitted, was used. Cysteine or sodium sulfide served as reducing agent and source of sulfur. Both strains grew in media lacking YE, CAS or any other source of amino acids (including cysteine)

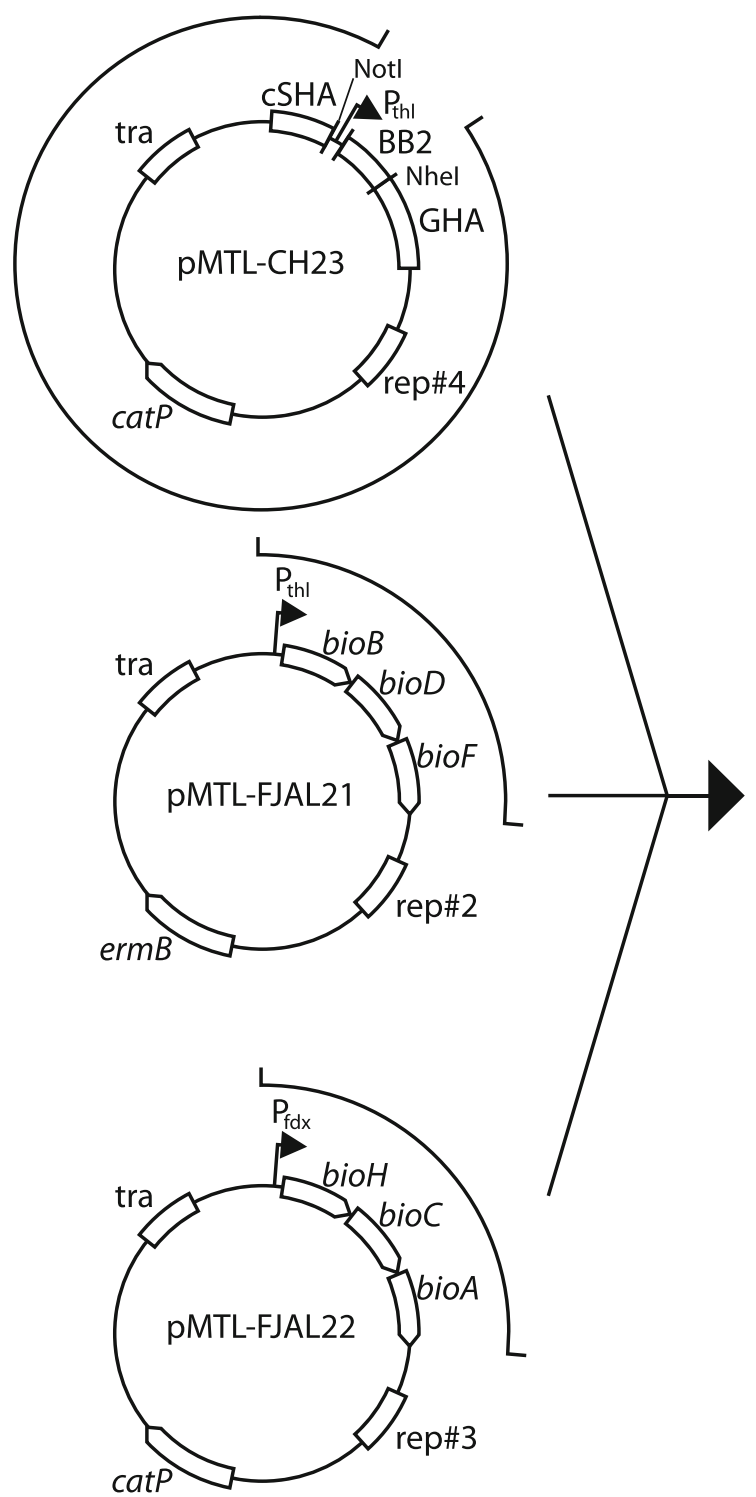

Fig. 2 Schematic representation of HiFi assembly of pMTL-FJAL23 integrative ACE vector. Full detail is provided in the text. The indicated sections of depicted plasmids were assembled. For pMTL-FJAL21 and pMTL-FJAL22, the sections were amplified by PCR with overlapping when B-vitamins were supplemented (Table 1). Auxotrophy for vitamins was initially tested in MBM + CAS from which one vitamin was omitted, for each vitamin. Growth was not observed in serial dilutions in medium lacking pantothenate, biotin or thiamine. In further experiments in MBM medium with sodium sulfide, growth was only obtained if pantothenate, biotin and thiamine were present (Table 1). The outcome of growth experiments with wild type, and also the derived strains mentioned below, was the same if fructose or $\mathrm{CO}$ was used as growth substrate and no obvious differences in cell morphology was observed by phase contrast microscopy. Also, the final optical densities of cultures that grew were very similar (data not shown), either due to another compound in

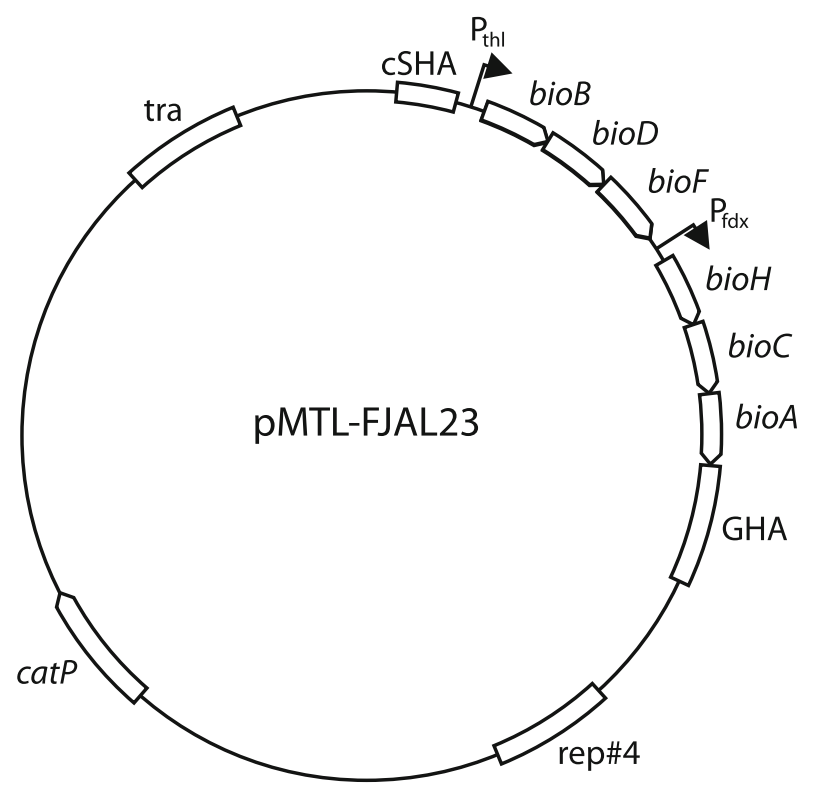

primers. The section forming the backbone of pMTL-CH23 was obtained after digestion of the plasmid with NotI and NheI. Sizes of depicted molecules do not relate to molecular size 
the medium than $\mathrm{CO}$ or fructose being growth limiting, or due to the limitations of the used spectrophotometric technique that did not allow dilution of the samples.

\section{Metabolic pathway analysis}

Bioinformatic analysis was used to investigate whether incomplete metabolic pathways could account for auxotrophy in C. autoethanogenum and C. ljungdahlii. It was found that the whole genome metabolic models for both strains, iCLAU786 and iHN637, respectively, contained functional pathways to pantothenate and iCLAU786 also to thiamine. This was in disagreement with our growth experiments. Reconstruction of metabolic pathways for pantothenate, biotin and thiamine was therefore performed by manual curation of whole genome sequences and revealed that one or more genes were missing for each biosynthetic pathway and that these deficiencies were the same in both microorganisms. These pathways are discussed in more detail in "Discussion"; here, we mention only the affected steps. The pantothenate biosynthesis pathway lacked orthologs for panB, panC and panD (Fig. 3a). Of the biotin biosynthesis pathway, only bio $B$ was present and no orthologs for bioW, bioI, bioD, bioF, bioH, bio $C$ and bioH were found (Fig. 3b). The thiamine biosynthesis pathway lacked orthologs for thiG, thiH and thiC (Fig. 3c). In addition, no clear ortholog for TenI was present in the thiamine biosynthesis pathway. Two putative orthologs for B. subtilis TenI, annotated as thiE1 and thiE2, contained relative poor query coverage ( $49 \%$ and $67 \%$ ) and sequence identity $(48 \%$ and $31 \%)$ as obtained with BLAST. While genes annotated as thiS and thiF were present, however only part of the genes showed homology with known thiS and thiF genes. Gene context of thiS and thiF genes did not directly hint at thiamine biosynthesis. Introduction of the steps that were missing in the vitamin biosynthesis pathways of the metabolic models iCLAU786 and iHN637 resulted in the production of pantothenate, thiamine and biotin by these models (Online Resource 1).

\section{Genetic engineering of pantothenate prototrophy}

C. acetobutylicum, as a phylogenetically closely related microorganism, was used as source for the genes needed to introduce pantothenate prototrophy in C. autoethanogenum and C. ljungdahlii. Modular pMTL80000 vectors with $\mathrm{P}_{\mathrm{fdx}}$ or $\mathrm{P}_{\mathrm{thl}}$ promoter (Heap et al. 2009) and downstream panB, panC and/ or panD genes of $C$. acetobutylicum were created (Table 3 ) and introduced into $C$. autoethanogenum and $C$. ljungdahlii to test whether various combinations of these genes could confer pantothenate prototrophy on the target microorganisms. Plasmid-bearing strains were inoculated in medium lacking pantothenate to test their viability. Strains that harboured a plasmid that carried all three genes were able to grow in medium lacking pantothenate (Table 4). This plasmid was maintained for at least eight serial transfers in medium without antibiotic. Also, strains that carried two plasmids that together specified all three genes were able to grow in medium without pantothenate and were maintained for at least eight serial transfers in absence of antibiotic (Table 4). It was also of interest to see whether pantolactone and beta-alanine could serve as provitamins and replace the need for panB or panD, respectively (Fig. 1a). Growth was detected for plasmid-bearing strains that possessed panC in medium that contained pantolactone and beta-alanine, and in those strains that carried panB and $p a n C$ in medium supplemented with pantolactone, as well as in cells carrying panC and panD in medium containing beta-alanine (Table 4).

\section{Chromosomal integration of panBCD}

A partial pyrE deletion mutant of $C$. autoethanogenum and C. ljungdahlii was created and then used to integrate pan $B C D$ genes into the chromosome using ACE, concomitant with restoration of uracil prototrophy. Integrants of both species designated as strains PANTS were able to grow without pantothenate supplementation of the medium (Table 1, Table 4).

\section{Genetic engineering of biotin prototrophy}

Initially, C. acetobutylicum was used as a potential source of the genes needed to confer biotin prototrophy on C. autoethanogenum and C. ljungdahlii. C. acetobutylicum has been reported to grow in medium without biotin supplemented (Li et al. 2013), but only contains genes for BioB, BioA, BioD and BioY (Yang et al. 2016).

However, overexpression of bioYDA significantly improved its growth (Yang et al. 2016). Accordingly, an expression vector was created that carried the bioYDA genes of C. acetobutylicum (CA_c1360-c1362) and introduced into C. autoethanogenum by conjugative plasmid transfer. However, wild-type and transconjugant strains of C. autoethanogenum were not able to grow in medium without biotin. If pimelate was added to the medium as a provitamin, then the transconjugant strains were able to grow, but not the wild-type strains (Table 4).

The strict anaerobic, Gram-positive, carboxydotrophic bacterium Desulfotomaculum nigrificans strain CO-1SRB was used as source for genes to introduce a complete biosynthesis pathway for biotin into C. autoethanogenum. The needed bio genes for biotin biosynthesis in this strain were conveniently located in a single operon bioBDFHCA (Desca_0053-0058). Two expression vectors were created that carried bioBDF downstream of $\mathrm{P}_{\text {thl }}$ (pMTL-FJAL21) and bioHCA downstream of $\mathrm{P}_{\mathrm{fdx}}$ (pMTL-FJAL22). Both vectors were introduced into $C$. autoethanogenum by 
conjugative plasmid transfer, involving the use of two $E$. coli donors carrying one or other of the plasmids. It has to be noted that the success rate for this simultaneous conjugation was low and only resulted in a few colonies in the third attempt. Transconjugants were able to grow in medium without biotin (Table 4).

For chromosomal integration of all the biotin biosynthesis genes, a single integration vector was created using NEB HiFi assembly and plasmids pMTL-FJAL21, pMTL-FJAL22 and pMTL-CH23 as templates. A single plasmid avoids the need for multiple iterations of ACE, although its larger size is likely to result in lower rates of plasmid transfer. Nonetheless, transconjugants were successfully isolated for $C$. autoethanogenum in which integration had taken place and this strain was designated strain BIO. Moreover, these integrants were able to grow on medium without biotin supplementation (Table 4).

\section{Combined pantothenate and biotin prototrophy in $C$. autoethanogenum}

An expression vector pMTL-FJAL24 that carried the $\mathrm{P}_{\text {thl }}{ }^{-}$ bioBDF- $\mathrm{P}_{\mathrm{fdx}}-$-bioHCA module was created and transferred into $C$. autoethanogenum by conjugation simultaneously with pMTL-FJAL3 that carried $\mathrm{P}_{\text {thl-panBCD. Cells carrying these }}$ two plasmids were able to grow in medium without biotin and pantothenate supplementation, although the observed growth rate was reduced. This strain was designated $C$. autoethanogenum biopants (Table 1, Table 4).

\section{Genetic engineering of thiamine prototrophy}

Comparative genomics revealed that in contrast to C. autoethanogenum and C. ljungdahlii, a thiC gene is present in the closely related acetogenic species $C$. ragsdalei, with directly downstream a $p u r F$ gene (Bengelsdorf et al. 2016). PurF is a phosphoribosyl pyrophosphate (PRPP) amidotransferase that synthesises phosphoribosylamine (PRA), the first step in purine biosynthesis and a precursor of aminoimidazole ribotide (AIR), the substrate of ThiC. Despite the presence of purF in the genomes of C. autoethanogenum and C. ljungdahlii, and potentiality of alternative paths to AIR (Bazurto et al. 2016), purF was included in the plasmid constructed to complement thiamine biosynthesis. A vector with the thiC-purF genes and the directly upstream intergenic region, to include the native promoter, was constructed (pBAS001/pMTL8524-thiC-purF) and introduced into $C$. autoethanogenum and C. ljungdahlii. Transformants were able to grow in medium without thiamine in successive transfers, while growth of the wild-type and plasmid control strain ceased after two subculture steps.

\section{Discussion}

Growth experiments indicated that C. autoethanogenum and C. ljungdahlii are auxotrophic for pantothenate, biotin and thiamine. Also, genomic analysis revealed that both microorganisms lacked the same genes in their biosynthetic pathways. Complementation of pantothenate (in C. ljungdahlii and C. autoethanogenum), biotin (in C. autoethanogenum) and thiamine (in C. ljungdahlii and C. autoethanogenum) biosynthetic pathways is discussed.

\section{Pantothenate biosynthesis}

Pantothenate is a key precursor for the synthesis of coenzyme A $(\mathrm{CoA})$ and produced from aspartate and 2-ketovaline, an intermediate of the valine biosynthesis pathway, in four enzymatic steps catalysed by PanB, C, D and E (Fig. 3a) (Leonardi and Jackowski 2007). The pantothenate biosynthesis pathway of C. autoethanogenum and C. ljungdahlii lacked genes for ketopantoate hydroxymethyltransferase (PanB), pantothenate synthetase (PanC), and aspartate carboxylase (PanD). The panBCD gene cluster of C. acetobutylicum (CA_C2914-2916) was successfully used to complement the pantothenate biosynthesis pathway of $C$. ljungdahlii and C. autoethanogenum, from plasmid and when integrated in the genome. Transformations with single genes and pairs of genes demonstrated that pantolactone could substitute for $\mathrm{pan} B$ and beta-alanine for $p a n D$. If pantolactone and beta-alanine were supplied simultaneously, only panC was needed in addition for growth in the absence of pantothenate from the medium. Since yeast extract does not contain sufficient pantothenate to support growth of C. autoethanogenum and C. ljungdahlii, the pantothenate biosynthesis genes can be used as selectable marker in media that lack pantothenate, but contain yeast extract to promote rate of colony formation. Additionally, the three genes can be used independently in combination with beta-alanine and/or pantolactone to allow for cloning strategies that require sequential transfers of multiple vectors.

\section{Biotin biosynthesis}

Of the biotin biosynthetic pathway, only bioB and bio $Y$ were present (Fig. 3b). BioB catalyses the conversion of desthiobiotin to biotin, the final step in biotin biosynthesis. BioY is a biotin transporter. Genes for conversion of the pimeloyl moiety to biotin (bioD, bioA and bioF), which are conserved across all domains of life, were not found. Also, dedicated genes for the synthesis of the pimeloyl moiety (bioW and bioI or bioH and bioC) were absent (Fig. 1b). The steps for the conversion of the pimeloyl-moiety $\mathrm{BioC}$ and $\mathrm{BioH}$ are key enzymes in the biotin synthesis pathway of E. coli, which "hijacks" the fatty acid biosynthesis cascade to elongate the carbon chain of acyl-carrier protein ([acp]) 

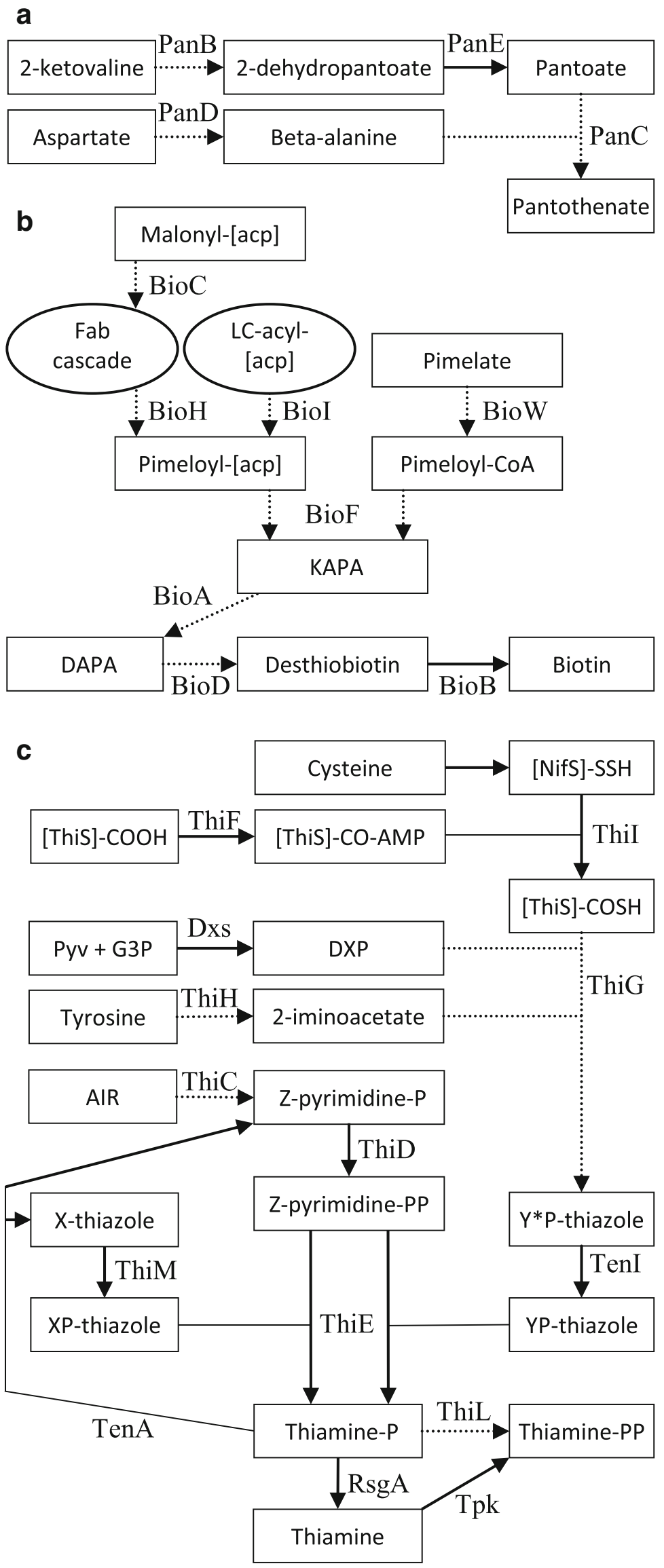
Fig. 3 The incomplete pantothenate, biotin and thiamine biosynthesis pathways of $C$. autoethanogenum and C. ljungdahlii. Missing steps are indicated by a dotted line. Details on enzymes are provided in the text. a Pantothenate biosynthesis pathway. b Biotin biosynthesis pathway. c Thiamine biosynthesis pathway. Abbreviations: [acp] = acyl-carrier protein; $\mathrm{Fab}=$ fatty acid biosynthesis; $\mathrm{LC}=$ long chain; $\mathrm{KAPA}=8$ amino-7-oxononanoate; DAPA = 7,8-diaminononanoate; Pyv = pyruvate; G3P = glyceraldehyde-3-phosphate; DXP = 1-deoxy-Dxylulose 5-phosphate; AIR = aminoimidazole ribotide; $X=4$-methyl-5[(2-hydroxy)-ethyl]-2,5-dihydro-; XP = 4-methyl-5-[(2-phosphonooxy)ethyl]-2,5-dihydro-; YP = 2-carboxy-4-methyl-5-[(2-phosphonooxy)ethyl]-2,5-dihydro-; $Y * P=2$-carboxy-4-methyl-5-[(2-phosphonooxy)ethylidene ]-2,5-dihydro-; $Z=4$-amino-2-methyl-5(diphosphooxymethyl)-

bound malonyl methyl ester that is formed by BioC (Lin et al. 2011). BioH salvages pimeloyl-[acp] methyl ester from the cascade and converts it to pimeloyl-[acp]. BioI and BioW are used by $B$. subtilis for biosynthesis of the pimeloyl moiety of biotin (Manandhar and Cronan 2017). BioI utilises molecular oxygen to cleave pimeloyl-acp from long chain acyl-[acp] molecules and is as such unsuitable for use in an anaerobe. While bioI was shown not essential in B. subtilis, its deletion resulted in a biotin bradytroph. BioW was reported essential in
B. subtilis and converts pimelate to pimeloyl-CoA (Manandhar and Cronan 2017). Furthermore, it was found that BioF of B. subtilis specifically used pimeloyl-CoA, whereas E. coli BioF also used pimeloyl-[acp] (Manandhar and Cronan 2018). Recently, it was reported that C. acetobutylicum was able to grow in defined medium without biotin (Li et al. 2013; Yang et al. 2016), although carryover of biotin in the method used cannot be excluded. Only bioB, bio $Y$, bioD and bioA were identified in its genome and not bioW, bioI or bioF (Yang et al. 2016). We were also unable to identify bioC and bioH (data not shown). Accordingly, the bioYDA gene cluster of C. acetobutylicum was introduced into C. autoethanogenum. The transconjugants obtained were not, however, prototrophic for biotin. Transconjugants carrying the bioYDA genes did grow when pimelic acid was added to medium lacking biotin, while wild-type $C$. autoethanogenum did not grow. Apparently, the gene cluster bioYDA was sufficient for $C$. autoethanogenum to convert pimelic acid to biotin, despite the absence of known genes for BioW and BioF. It remains to be investigated whether $C$. autoethanogenum contains alternative genes to catalyse these steps or that 7-keto-8aminopelargonic acid (KAPA), the substrate of BioA, is

Table 4 Growth of recombinant strains in defined media

\begin{tabular}{|c|c|c|c|c|c|c|}
\hline \multirow[t]{2}{*}{ Genotype } & \multirow[t]{2}{*}{ Recombinant molecule } & \multicolumn{5}{|l|}{ Medium } \\
\hline & & Supplement & MBM & MBM-P & MBM-B & MBM-T \\
\hline $\mathrm{Wt}$ & & & + & - & - & - \\
\hline $\operatorname{pan} B C D$ & pMTL-FJAL3 & & + & + & & \\
\hline $\operatorname{pan} B C D$ & $\mathrm{C}$ & & + & + & & \\
\hline $\operatorname{pan} B$ & pMTL-FJAL6 & & + & - & & \\
\hline panC & pMTL-FJAL7 & & + & - & & \\
\hline panD & pMTL-FJAL8 & & + & - & & \\
\hline $\operatorname{pan} B C$ & pMTL-FJAL5 & & + & - & & \\
\hline panCD & pMTL-FJAL4 & & + & - & & \\
\hline $\operatorname{pan} B C+\operatorname{pan} D$ & pMTL-FJAL5 + pMTL-FJAL8 & & + & + & & \\
\hline $\operatorname{pan} C D+\operatorname{pan} B$ & pMTL-FJAL4 + pMTL-FJAL6 & & + & + & & \\
\hline $\operatorname{pan} B C$ & pMTL-FJAL5 & b-alanine & + & + & & \\
\hline $\operatorname{pan} C D$ & pMTL-FJAL4 & pantolactone & + & + & & \\
\hline $\operatorname{panC}$ & pMTL-FJAL7 & b-alanine + pantolactone & + & + & & \\
\hline bioYDA & pMTL-FJAL20 & & + & & - & \\
\hline bioYDA & pMTL-FJAL20 & Pimelate & + & & + & \\
\hline bioBDF & pMTL-FJAL21 & & + & & - & \\
\hline bioHCA & pMTL-FJAL22 & & + & & - & \\
\hline bioBDF + bioHCA & pMTL-FJAL21 + pMTLFJAL22 & & + & & + & \\
\hline bioBDF_bioHCA & pMTL-FJAL24 & & + & & + & \\
\hline bioBDF_bioHCA & $\mathrm{C}$ & & + & & + & \\
\hline panBCD+bioBDF_bioHCA & pMTL-FJAL3+ pMTL-FJAL24 & & + & + & + & \\
\hline thiC-purF & pMTL-BAS1 & & + & & & + \\
\hline
\end{tabular}

$c$ chromosome, $P$ except pantothenate, $B$ except biotin, $T$ except thiamine 
formed from pimelic acid through a completely different mechanism (Fig. 1b). Full biotin prototrophy of C. autoethanogenum was obtained after transformation with gene clusters bioBDF and bioHCA of the Gram-positive, strictly anaerobic, carboxydotrophic $D$. nigrificans CO-1SRB. These genes also conveyed biotin prototrophy when integrated in the genome (Table 4).

Prototrophy for pantothenate and biotin was combined successfully by transfer of plasmids carrying panBCD and bioBDF-bioHCA gene clusters to $C$. autoethanogenum. Growth of transconjugants was noticeably slower (data not shown) and likely due to the need for the cells to maintain two plasmids of which one of considerable size $(\sim 13 \mathrm{kbp})$. Transfer of both plasmids was unsuccessful in C. ljungdahlii and most likely due to the low efficiency of the used transformation method. All generated prototroph strains were also able to grow with $\mathrm{CO}$ as sole carbon and energy source (instead of fructose).

\section{Thiamine biosynthesis}

Thiamine biosynthesis follows a complex pathway in which a pyrimidine and a thiazole moiety are joined to form thiamine pyrophosphate (TPP), the active form of thiamine, and requires at least 10 enzymes in anaerobic bacteria (Fig. 3). The thiazole is formed through activity of ThiFSGH, Thil and Dxs and the pyrimidine through ThiC and ThiD. The pyrimidine and thiazole are coupled by ThiE to form thiamine monophosphate (TMP) and in E. coli is phosphorylated to TPP by ThiL (Begley1999). In some microorganisms, ThiL is lacking and instead TMP is first dephosphorylated by RgsA to thiamine, that in turn is converted by thiamine pyrophosphokinase (Tpk) to TPP. Additional enzymes associated with the thiamine biosynthesis pathway exist that play a role in thiamine salvage pathway (TenA and ThiM) or that convert (by TenI) the thiazole ( $\mathrm{Y}^{*} \mathrm{P}$-thiazole) produced by thiazole synthase (ThiG) to a tautomer (YP-thiazole) that is more readily used by thiamine phosphate synthase (ThiE) (Hazra et al. 2009, 2011). The thiamine biosynthesis pathway of C. autoethanogenum and C. ljungdahlii was found to lack genes for the key enzymes 2-iminoacetate synthase (ThiH), ThiG and phosphomethylpyrimidine synthase (ThiC) (Fig. 3c). Two paralogs for ThiE were found, ThiE1 and ThiE2, and it is likely one of these functions as TenI (Rodionov et al. 2002). The lack of thiC, thiG and thiH is common among enteric Clostridia and instead an operon with thiamine salvage pathway genes and transporters for the pyrimidine and thiazole moieties may be present (Rodionov et al. 2002), as was the case for C. autoethanogenum and C. ljungdahlii that contained a ThiDME operon, a transporter for the thiamine (ThiW) and pyrimidine (ThiYXZ) moiety, and must contain an uptake system for thiamine as the wild-type strains are able to grow in minimal medium with thiamine supplemented.

However, we found that the thiamine biosynthesis pathway could be completed with just the thiC-purF operon from $C$. ragsdalei in minimal medium that lacked thiamine and precursors of the pyrimidine and thiazole moieties. This indicates that either unknown genes or an unknown pathway exists that replaces ThiG and ThiH function.

C. autoethanogenum and C. ljungdahlii were found to be auxotrophic for pantothenate, biotin and thiamine. Suitable genes to complement biosynthesis pathways were identified and successfully used to confer prototrophy for each of these three vitamins in vivo. This is the first report of chromosomal integration of genes in the chromosomes of C. autoethanogenum and C. ljungdahlii by ACE (Heap et al. 2012). Study of the biosynthetic pathways for these vitamins revealed that the available whole genome metabolic models iHN637 and iCLAU786 (Nagarajan et al. 2013; Marcellin et al. 2016) erroneously already contained pathways for pantothenate and thiamine production. The models used automatically annotated genome sequences that were not manually curated. Interestingly, iCLAU786 produced pantothenate with just panBC while we showed in vivo that panD was essential as well. The beta-alanine that is produced by panD was in the model derived from uracil (data not shown). Both models were amended with complete pathways to reflect the prototrophy for pantothenate, biotin and thiamine (Online Resource 1), however with the thiamine biosynthesis pathway as it appears in iCLAU786 and that includes a ThiGH thiazole synthase reaction. In reality, it remains unclear how the thiazole is formed as we found genes for ThiG and ThiH to be lacking from the genome sequences and only introduction of thiC and purF resulted in complementation of thiamine biosynthesis. Similarly, biotin could be synthesised from pimelate without the apparent need of bioW and bioF. Likely, these metabolic steps are carried by still unidentified genes that are present in these strict anaerobic Gram-positive acetogens.

Acknowledgements This work was supported by the Biotechnology and Biological Sciences Research Council [grant numbers BB/L502030/1, $\mathrm{BB} / \mathrm{K} 00283 \mathrm{X} / 1$ ] as part of the BBSRC Longer and Larger Grant GASCHEM; and the industrial partner LanzaTech Inc.

Funding This work was supported by the Biotechnology and Biological Sciences Research Council [grant numbers BB/L502030/1, BB/ K00283X/1] as part of the BBSRC Longer and Larger Grant GASCHEM; and the industrial partner LanzaTech Inc. 


\section{Compliance with ethical standards}

Conflict of interest FJA declares that she has no conflict of interest. BAS declares that he has no conflict of interest.

$\mathrm{CMH}$ declares that he has no conflict of interest.

$\mathrm{RN}$ declares that he has no conflict of interest.

$\mathrm{KW}$ declares that he has no conflict of interest.

$\mathrm{KM}$ is employed by LanzaTech. LanzaTech has commercial interest in gas fermentation.

SDS is employed by LanzaTech. LanzaTech has commercial interest in gas fermentation.

NPM declares that he has no conflict of interest.

AMH declares that he has no conflict of interest.

Ethical approval This article does not contain any studies with human participants or animals performed by any of the authors.

Open Access This article is distributed under the terms of the Creative Commons Attribution 4.0 International License (http:// creativecommons.org/licenses/by/4.0/), which permits unrestricted use, distribution, and reproduction in any medium, provided you give appropriate credit to the original author(s) and the source, provide a link to the Creative Commons license, and indicate if changes were made.

\section{References}

Abrini J, Naveau H, Nyns E-J (1994) Clostridium autoethanogenum, sp. nov., an anaerobic bacterium that produces ethanol from carbon monoxide. Arch Microbiol 161:345-351. https://doi.org/10.1007/ BF00303591

Agarwala R, Barrett T, Beck J, Benson DA, Bollin C, Bolton E, Bourexis D, Brister JR, Bryant SH, Canese K, Charowhas C, Clark K, DiCuccio M, Dondoshansky I, Feolo M, Funk K, Geer LY, Gorelenkov V, Hlavina W, Hoeppner M, Holmes B, Johnson M, Khotomlianski V, Kimchi A, Kimelman M, Kitts P, Klimke W, Krasnov S, Kuznetsov A, Landrum MJ, Landsman D, Lee JM, Lipman DJ, Lu Z, Madden TL, Madej T, Marchler-Bauer A, Karsch-Mizrachi I, Murphy T, Orris R, Ostell J, O'Sullivan C, Palanigobu V, Panchenko AR, Phan L, Pruitt KD, Rodarmer K, Rubinstein W, Sayers EW, Schneider V, Schoch CL, Schuler GD, Sherry ST, Sirotkin K, Siyan K, Slotta D, Soboleva A, Soussov V, Starchenko G, Tatusova TA, Todorov K, Trawick BW, Vakatov D, Wang Y, Ward M, Wilbur WJ, Yaschenko E, Zbicz K (2017) Database resources of the National Center for biotechnology information. Nucleic Acids Res 45:D12-D17. https://doi.org/10.1093/ nar/gkw1071

Aghbashlo M, Tabatabaei M, Dadak A, Younesi H, Najafpour G (2016) Exergy-based performance analysis of a continuous stirred bioreactor for ethanol and acetate fermentation from syngas via WoodLjungdahl pathway. Chem Eng Sci 143:36-46. https://doi.org/10. 1016/J.CES.2015.12.013

Altschul SF, Gish W, Miller W, Myers EW, Lipman DJ (1990) Basic local alignment search tool. J Mol Biol 215:403-410. https://doi.org/10. 1016/S0022-2836(05)80360-2

Bateman A, Martin MJ, O’Donovan C, Magrane M, Alpi E, Antunes R, Bely B, Bingley M, Bonilla C, Britto R, Bursteinas B, Bye-AJee H, Cowley A, Da Silva A, De Giorgi M, Dogan T, Fazzini F, Castro LG, Figueira L, Garmiri P, Georghiou G, Gonzalez D, Hatton-Ellis E, Li W, Liu W, Lopez R, Luo J, Lussi Y, MacDougall A, Nightingale A, Palka B, Pichler K, Poggioli D, Pundir S, Pureza L, Qi G, Rosanoff S, Saidi R, Sawford T, Shypitsyna A, Speretta E, Turner E, Tyagi N, Volynkin V, Wardell T, Warner K, Watkins X,
Zaru R, Zellner H, Xenarios I, Bougueleret L, Bridge A, Poux S, Redaschi N, Aimo L, ArgoudPuy G, Auchincloss A, Axelsen K, Bansal P, Baratin D, Blatter MC, Boeckmann B, Bolleman J, Boutet E, Breuza L, Casal-Casas C, De Castro E, Coudert E, Cuche B, Doche M, Dornevil D, Duvaud S, Estreicher A, Famiglietti L, Feuermann M, Gasteiger E, Gehant S, Gerritsen V, Gos A, GruazGumowski N, Hinz U, Hulo C, Jungo F, Keller G, Lara V, Lemercier P, Lieberherr D, Lombardot T, Martin X, Masson P, Morgat A, Neto T, Nouspikel N, Paesano S, Pedruzzi I, Pilbout S, Pozzato M, Pruess M, Rivoire C, Roechert B, Schneider M, Sigrist C, Sonesson K, Staehli S, Stutz A, Sundaram S, Tognolli M, Verbregue L, Veuthey AL, Wu CH, Arighi CN, Arminski L, Chen C, Chen Y, Garavelli JS, Huang H, Laiho K, McGarvey P, Natale DA, Ross K, Vinayaka CR, Wang Q, Wang Y, Yeh LS, Zhang J (2017) UniProt: the universal protein knowledgebase. Nucleic Acids Res 45:D158D169. https://doi.org/10.1093/nar/gkw1099

Bazurto JV, Farley KR, Downs DM (2016) An unexpected route to an essential cofactor: Escherichia coli relies on threonine for thiamine biosynthesis. MBio 7:1-9. https://doi.org/10.1128/mBio.01840-15

Bengelsdorf FR, Poehlein A, Linder S, Erz C, Hummel T, Hoffmeister S, Daniel R, Dürre P (2016) Industrial Acetogenic biocatalysts: a comparative metabolic and genomic analysis. Front Microbiol 7:1036. https://doi.org/10.3389/fmicb.2016.01036

Brown SD, Nagaraju S, Utturkar S, De Tissera S, Segovia S, Mitchell W, Land ML, Dassanayake A, Köpke M (2014) Comparison of singlemolecule sequencing and hybrid approaches for finishing the genome of Clostridium autoethanogenum and analysis of CRISPR systems in industrial relevant Clostridia. Biotechnol Biofuels 7:40. https://doi.org/10.1186/1754-6834-7-40

Bruno-Barcena JM, Chinn MS, Grunden AM (2013) Genome sequence of the autotrophic acetogen Clostridium autoethanogenum JA1-1 strain DSM 10061, a producer of ethanol from carbon monoxide. Genome Announc 1:e00628-e00613. https://doi.org/10.1128/ genomeA.00628-13

Dadak A, Aghbashlo M, Tabatabaei M, Younesi H, Najafpour G (2016) Using exergy to analyse the sustainability of fermentative ethanol and acetate production from syngas via anaerobic bacteria (Clostridium ljungdahlii). Sustain Energy Technol Assess 15:1119. https://doi.org/10.1016/j.seta.2016.03.001

Dürre P (2017) Gas fermentation - a biotechnological solution for today's challenges. Microb Biotechnol 10:14-16. https://doi.org/10.1111/ 1751-7915.12431

Hazra A, Chatterjee A, Begley TP (2009) Biosynthesis of the Thiamin Thiazole in : Identification of the Product of the Thiazole SynthaseCatalyzed Reaction. Journal of the American Chemical Society 131 (9):3225-3229

Hazra AB, Han Y, Chatterjee A, Zhang Y, Lai R-Y, Ealick SE, Begley TP (2011) A Missing Enzyme in Thiamin Thiazole Biosynthesis: Identification of TenI as a Thiazole Tautomerase. Journal of the American Chemical Society 133 (24):9311-9319

Heap JT, Ehsaan M, Cooksley CM, Ng Y-K, Cartman ST, Winzer K, Minton NP (2012) Integration of DNA into bacterial chromosomes from plasmids without a counter-selection marker. Nucleic Acids Res 40:e59. https://doi.org/10.1093/nar/gkr1321

Heap JT, Pennington OJ, Cartman ST, Minton NP (2009) A modular system for Clostridium shuttle plasmids. J Microbiol Methods 78: 79-85. https://doi.org/10.1016/J.MIMET.2009.05.004

Humphreys CM, McLean S, Schatschneider S, Millat T, Henstra AM, Annan FJ, Breitkopf R, Pander B, Piatek P, Rowe P, Wichlacz AT, Woods C, Norman R, Blom J, Goesman A, Hodgman C, Barrett D, Thomas NR, Winzer K, Minton N (2015) Whole genome sequence and manual annotation of Clostridium autoethanogenum, an industrially relevant bacterium. BMC Genomics 16:1085. https://doi.org/ 10.1186/s12864-015-2287-5 
Humphreys CM, Minton NP (2018) Advances in metabolic engineering in the microbial production of fuels and chemicals from $\mathrm{C} 1$ gas. Biotechnol Curr Opin Biotechnol 50:174-181

Kanehisa M, Furumichi M, Tanabe M, Sato Y, Morishima K (2017) KEGG: new perspectives on genomes, pathways, diseases and drugs. Nucleic Acids Res 45:D353-D361. https://doi.org/10.1093/ nar/gkw1092

Knight T (2003) Idempotent vector design for standard assembly of biobricks. MIT synthetic biology working group technical reports. $<$ http://hdl.handle.net/1721.1/21168> (2003) Accessed Aug 2018

Köpke M, Held C, Hujer S, Liesegang H, Wiezer A, Wollherr A, Ehrenreich A, Liebl W, Gottschalk G, Dürre P (2010) Clostridium ljungdahlii represents a microbial production platform based on syngas. Proc Natl Acad Sci 107:13087-13092. https://doi.org/10. 1073/pnas. 1004716107

Leonardi R, Jackowski S (2007) Biosynthesis of Pantothenic Acid and Coenzyme A. EcoSal Plus 2 (2)

Li Z, Xiao H, Jiang W, Jiang Y, Yang S (2013) Improvement of solvent production from xylose mother liquor by engineering the xylose metabolic pathway in Clostridium acetobutylicum EA 2018. Appl Biochem Biotechnol 171:555-568. https://doi.org/10.1007/s12010013-0414-9

Liew F, Henstra AM, Köpke M, Winzer K, Simpson SD, Minton NP (2017) Metabolic engineering of Clostridium autoethanogenum for selective alcohol production. Metab Eng 40:104-114. https:// doi.org/10.1016/j.ymben.2017.01.007

Liew F, Martin ME, Tappel RC, Heijstra BD, Mihalcea C, Köpke M (2016) Gas fermentation - a flexible platform for commercial scale production of low-carbon-fuels and chemicals from waste and renewable feedstocks. Front Microbiol 7:694. https://doi.org/10.3389/ fmicb.2016.00694

Lin S, Hanson RE, Cronan JE (2011) Biotin synthesis begins by hijacking the fatty acid synthetic pathway. Nat Chem Biol 6:682-688. https:// doi.org/10.1038/nchembio.420.Biotin

Manandhar M, Cronan JE (2017) Pimelic acid, the first precursor of the Bacillus subtilis biotin synthesis pathway, exists as the free acid and is assembled by fatty acid synthesis. Mol Microbiol 104:595-607. https://doi.org/10.1111/mmi.13648

Manandhar M, Cronan JE (2018) A canonical biotin synthesis enzyme, 8Amino-7-Oxononanoate synthase (BioF), utilizes different acyl Chain donors in Bacillus subtilis and Escherichia coli. Appl Environ Microbiol 84:e02084-e02017. https://doi.org/10.1128/ AEM.02084-17

Marcellin E, Behrendorff JB, Nagaraju S, DeTissera S, Segovia S, Palfreyman R, Daniell J, Licona-Cassani C, Quek L, Speight R, Hodson MP, Simpson SD, Mitchell WP, Köpke M, Nielsen LK (2016) Low carbon fuels and commodity chemicals from waste gases - systematic approach to understand energy metabolism in a model acetogen. Green Chem 1:3020-3028. https://doi.org/10. 1039/C5GC02708J

Minton NP, Ehsaan M, Humphreys CM, Little GT, Baker J, Henstra AM, Liew F, Kelly ML, Sheng L, Schwarz K, Zhang Y (2016) A roadmap for gene system development in Clostridium. Anaerobe 41:104-112. https://doi.org/10.1016/j.anaerobe.2016.05.011
Nagarajan H, Sahin M, Nogales J, Latif H, Lovley DR, Ebrahim A, Zengler K (2013) Characterizing acetogenic metabolism using a genome-scale metabolic reconstruction of Clostridium ljungdahlii. Microb Cell Factories 12:118. https://doi.org/10.1186/1475-2859$12-118$

Parshina SN, Sipma J, Nakashimada Y, Henstra AM, Smidt H, Lysenko AM, Lens PNL, Lettinga G, Stams AJM (2005) Desulfotomaculum carboxydivorans sp. nov., a novel sulfate-reducing bacterium capable of growth at 100\% CO. Int J Syst Evol Microbiol 55(Pt 5):2159 2165. https://doi.org/10.1099/ijs.0.63780-0

Pronk JT (2002) Auxotrophic yeast strains in fundamental and applied research. Appl Environ Microbiol 68:2095-2100. https://doi.org/10. 1128/AEM.68.5.2095

Purdy D, O'Keeffe TA, Elmore M, Herbert M, McLeod A, BokoriBrown M, Ostrowski A, Minton NP (2002) Conjugative transfer of clostridial shuttle vectors from Escherichia coli to Clostridium difficile through circumvention of the restriction barrier. Mol Microbiol 46:439-452. https://doi.org/10.1046/j.1365-2958.2002. 03134.x

Rajagopalan SP, Datar R, Lewis RS (2002) Formation of ethanol from carbon monoxide via a new microbial catalyst. Biomass Bioenergy 23:487-493. https://doi.org/10.1016/S0961-9534(02)00071-5

Rodionov DA, Vitreschak AG, Mironov AA, Gelfand MS (2002) Comparative genomics of thiamin biosynthesis in procaryotes. New genes and regulatory mechanisms. J Biol Chem 277:48949 48959. https://doi.org/10.1074/jbc.M208965200

Stams AJM, Van Dijk JB, Dijkema C, Plugge CM (1993) Growth of syntrophic propionate-oxidizing bacteria with fumarate in the absence of methanogenic bacteria. Appl Environ Microbiol 59:1114 1119

Tanner RS, Miller LM, Yang D (1993) Clostridium ljungdahlii sp-Nov, an Acetogenic species in Clostridial ribosomal-RNA homology group-I. Int J Syst Bacteriol 43:232-236. https://doi.org/10.1099/ 00207713-43-2-232

Visser M, Parshina SN, Alves JI, Sousa DZ, Pereira IAC, Muyzer G, Kuever J, Lebedinsky AV, Koehorst JJ, Worm P, Plugge CM, Schaap PJ, Goodwin LA, Lapidus A, Kyrpides NC, Detter JC, Woyke T, Chain P, Davenport KW, Spring S, Rohde M, Klenk HP, Stams AJM (2014) Genome analyses of the carboxydotrophic sulfate-reducers Desulfotomaculum nigrificans and Desulfotomaculum carboxydivorans and reclassification of Desulfotomaculum caboxydivorans as a later synonym of Desulfotomaculum nigrificans. Stand Genomic Sci 9:655-675. https://doi.org/10.4056/sigs.4718645

Yang Y, Lang N, Yang G, Yang S, Jiang W, Gu Y (2016) Improving the performance of solventogenic clostridia by reinforcing the biotin synthetic pathway. Metab Eng 35:121-128. https://doi.org/10. 1016/J.YMBEN.2016.02.006

Publisher's note Springer Nature remains neutral with regard to jurisdictional claims in published maps and institutional affiliations. 